\title{
Effect of oxaliplatin plus 5-fluorouracil or capecitabine on circulating and imaging biomarkers in patients with metastatic colorectal cancer: a prospective biomarker study
}

Reem D. Mahmood ${ }^{1 *+}$ D, Danielle Shaw ${ }^{2 \dagger}$, Tine Descamps ${ }^{3}$, Cong Zhou ${ }^{3}$, Robert D. Morgan ${ }^{1,4}$, Saifee Mullamitha ${ }^{1}$, Mark Saunders ${ }^{1}$, Nerissa Mescallado ${ }^{1}$, Alison Backen ${ }^{1,4}$, Karen Morris ${ }^{3}$, Ross A. Little ${ }^{4}$, Susan Cheung ${ }^{4}$, Yvonne Watson ${ }^{4}$, James P. B. O'Connor ${ }^{1,4}$, Alan Jackson ${ }^{5}$, Geoff J. M. Parker ${ }^{5,6,7}$, Caroline Dive ${ }^{3}$ and Gordon C. Jayson ${ }^{1,4}$

\section{Abstract}

Background: Patients with metastatic colorectal cancer are treated with cytotoxic chemotherapy supplemented by molecularly targeted therapies. There is a critical need to define biomarkers that can optimise the use of these therapies to maximise efficacy and avoid unnecessary toxicity. However, it is important to first define the changes in potential biomarkers following cytotoxic chemotherapy alone. This study reports the impact of standard cytotoxic chemotherapy across a range of circulating and imaging biomarkers.

Methods: A single-centre, prospective, biomarker-driven study. Eligible patients included those diagnosed with colorectal cancer with liver metastases that were planned to receive first line oxaliplatin plus 5-fluorouracil or capecitabine. Patients underwent paired blood sampling and magnetic resonance imaging (MRI), and biomarkers were associated with progression-free survival (PFS) and overall survival (OS).

Results: Twenty patients were recruited to the study. Data showed that chemotherapy significantly reduced the number of circulating tumour cells as well as the circulating concentrations of Ang1, Ang2, VEGF-A, VEGF-C and VEGF-D from pre-treatment to cycle 2 day 2. The changes in circulating concentrations were not associated with PFS or OS. On average, the MRI perfusion/permeability parameter, $K^{\text {trans }}$, increased in response to cytotoxic chemotherapy from pre-treatment to cycle 2 day 2 and this increase was associated with worse OS (HR 1.099, $95 \% \mathrm{Cl} 1.01-1.20, p=0.025)$.

\footnotetext{
*Correspondence: reem.mahmood@nhs.net

${ }^{\dagger}$ Reem D. Mahmood and Danielle Shaw contributed equally to this work.

'Christie NHS Foundation Trust, Wilmslow Road, Withington, Manchester M20 4BX, UK

Full list of author information is available at the end of the article
}

C C The Author(s). 2021 Open Access This article is licensed under a Creative Commons Attribution 4.0 International License, which permits use, sharing, adaptation, distribution and reproduction in any medium or format, as long as you give appropriate credit to the original author(s) and the source, provide a link to the Creative Commons licence, and indicate if changes were made. The images or other third party material in this article are included in the article's Creative Commons licence, unless indicated otherwise in a credit line to the material. If material is not included in the article's Creative Commons licence and your intended use is not permitted by statutory regulation or exceeds the permitted use, you will need to obtain permission directly from the copyright holder. To view a copy of this licence, visit http://creativecommons.org/licenses/by/4.0/ The Creative Commons Public Domain Dedication waiver (http://creativecommons.org/publicdomain/zero/1.0/) applies to the data made available in this article, unless otherwise stated in a credit line to the data. 
(Continued from previous page)

Conclusions: In patients diagnosed with colorectal cancer with liver metastases, treatment with standard chemotherapy changes cell- and protein-based biomarkers, although these changes are not associated with survival outcomes. In contrast, the imaging biomarker, $K^{\text {trans }}$, offers promise to direct molecularly targeted therapies such as anti-angiogenic agents.

Keywords: Colorectal cancer, Angiogenesis, Biomarkers, Bevacizumab

\section{Background}

Colorectal cancer is the fourth most common cancer in the United Kingdom, with around 42,000 new cases diagnosed each year [1]. For patients presenting with metastatic disease, overall survival remains poor, with only around $10 \%$ alive 5 years after their diagnosis [2]. The management of patients with metastatic colorectal cancer has evolved over the past decade with the additional use of molecularly targeted therapies in combination with cytotoxic chemotherapy.

For patients diagnosed with colorectal cancer with liver metastases, first line standard cytotoxic chemotherapy includes: FOLFOX (5-fluorouracil/folinic acid plus oxaliplatin), FOLFIRI (5-fluorouracil/folinic acid plus irinotecan) or CAPOX (capecitabine plus oxaliplatin) [3, 4]. Targeted therapies against vascular endothelial growth factors (VEGF) e.g. bevacizumab, and epidermal growth factor receptors (EGFR) e.g. cetuximab or panitumumab, are also recommended for first line management of patients with metastatic colorectal cancer in combination with cytotoxic chemotherapy [3, 4]. Indeed, bevacizumab, cetuximab and panitumumab have been shown in randomised phase III trials to prolong progression-free survival (PFS) [5-13] and overall survival (OS) [5-7, 12]. Circulating biomarkers, such as CK18, have been shown to be predictive of prognosis and progression in colorectal cancer $[14,15]$. In addition, use of genetics can guide the use of EGFR treatment, with patients that are KRAS/BRAF mutant not benefitting from these drugs [16-18]. However, there are no validated circulating or imaging biomarkers to guide the use of VEGF and EGFR therapies, which are expensive and associated with toxicity.

Early phase trials have assessed the effect of traditional cytotoxic chemotherapy in combination with bevacizumab using magnetic resonance imaging (MRI) [19-22] and circulating biomarkers [23-25]. However, in order to better understand the data reported for combination therapy, the effects of cytotoxic chemotherapy alone need to be assessed as a control. In this study, MRI and blood-based biomarkers were investigated in patients undergoing standard cytotoxic chemotherapy. Data from this study may improve the understanding of the utility of these biomarkers for future trials incorporating molecularly targeted therapies.

\section{Methods}

This was a prospective, single-centre, biomarker-driven study recruiting patients that were treated at the Christie NHS Foundation Trust for colorectal cancer with liver metastases. Ethical approval was obtained from the local ethics committee (see Supplementary Information). All patients gave written informed consent to participate in the study.

\section{Study participants}

Eligible participants included those with histologicallyproven colorectal cancer; liver metastases measuring at least $30 \mathrm{~mm}$ in the longest axis; 18 years of age or older; a World Health Organization (WHO) performance status of 0 to 2; were planned to commence primary therapy with oxaliplatin plus 5-fluorouracil (5-FU) or capecitabine; white cell blood count $\geq 4 \times 10^{9} / 1$; platelet count $\geq 100 \times 10^{9} / \mathrm{l}$; serum total bilirubin concentration $\leq$ $1.5 \times$ upper limit of normal (ULN); serum alkaline phosphatase concentration $\leq 5 \times \mathrm{ULN}$ and; a calculated glomerular filtration rate $\geq 50 \mathrm{ml}$ per minute.

Patients were excluded if MRI was contra-indicated due to standard criteria relating to metal implants or allergy to MRI contrast; use of adjuvant chemotherapy within 12 months prior to study enrolment; a personal medical history including any non-colorectal malignancy within 5 years of study enrolment; concurrent use of other investigational medicinal product or; pregnant or breast-feeding women.

\section{Study drugs}

Patients were treated with either oxaliplatin plus 5-FU (oxaliplatin $85 \mathrm{mg} / \mathrm{m}^{2}$ of body surface area [BSA] plus folinic acid $350 \mathrm{mg}$ and 5 -FU $400 \mathrm{mg} / \mathrm{m}^{2}$ on day 1 followed by 5 -FU $2400 \mathrm{mg} / \mathrm{m}^{2}$ intravenous infusion [46 h] every two-week cycle) or oxaliplatin plus capecitabine (oxaliplatin $130 \mathrm{mg} / \mathrm{m}^{2}$ on day 1 and capecitabine 1000 $\mathrm{mg} / \mathrm{m}^{2}$ on day 1 to 14 every 3-week cycle) for a maximum of 6 cycles.

\section{Clinical endpoints}

Clinical endpoints included progression-free survival (PFS) and overall survival (OS). Progressive disease was defined as the time interval from the date of study registration to the date of either clinical or radiological 
progression or death. On imaging, progressive disease was measured using the response evaluation criteria in solid tumours (RECIST) version 1.1 [26]. OS was defined as the time interval from the date of study registration to the date of death. All patients were followed up until they reached the PFS efficacy endpoint; no censoring was present in the dataset.

Computed tomography (CT) was performed every 8 weeks as part of standard tumour assessment. As part of standard treatment, plasma carcinoembryonic antigen (CEA) and lactate dehydrogenase (LDH) concentrations were measured at the start of each cycle of chemotherapy. Both can be used to predict prognosis and response to treatment in metastatic colorectal cancer [27, 28].

\section{Biomarker schedule}

A detailed description of the methodology used for the imaging and circulating biomarkers is provided in the Supplementary information.

Study time points for dynamic contrast-enhanced MRI (DCE-MRI) and diffusion weighted MRI (DW-MRI) included pre-treatment, cycle 1 day 2 , cycle 1 day 8 , cycle 2 day 2 of chemotherapy and following 12 weeks of chemotherapy. At pre-treatment, MRI scans were carried out twice, at least $24 \mathrm{~h}$ apart, to determine the repeatability of the imaging biomarkers. Regions of interest (ROIs) within the liver were defined manually by a trained operator, in order to determine whole tumour volume (WTV) from $\mathrm{T}_{1}$ - and $\mathrm{T}_{2}$-weighted images as well as the DCE-MRI images. Parameters derived from DCE-MRI included the transfer coefficient $\left(K^{\text {trans }}\right)$, volume of extravascular extracellular space $\left(v_{\mathrm{e}}\right)$ and vascular plasma volume $\left(v_{\mathrm{p}}\right)$. For DWI-MRI, the apparent diffusion coefficient (ADC) was derived.

Blood samples for circulating tumour cells (CTCs) and a panel of plasma-derived circulating protein biomarkers were collected at the same time points as MRI including Ang2, VEGF-A, VEGF-C, VEGF-D, VEGFR1, VEGFR2, IL6, IL8, Tie2, KGF, PlGF, FGFb, HGF, PDGFbb, SDF1b, E-selectin, M65 and VCAM-1.

\section{Statistical analysis}

The target recruitment for the study was 20 patients. All biomarkers were assessed for normality and transformed when necessary. To identify whether biomarker concentrations changed significantly from pre-treatment to cycle 2 day 2, paired Student's t-tests were performed. Cycle 2 day 2 of chemotherapy was selected for significance testing in order to determine the early effects of cytotoxic chemotherapy. A correlation network analysis was performed to examine the relationship between multiple biomarkers without the requirement to conduct multiple sequential analyses $[23,25]$. This was done based on Pearson correlations and build from the qgraph package in $\mathrm{R}$.

Cox proportional hazard regression was used for survival analysis, respecting the proportionality and linearity assumptions. Kaplan Meier curves were constructed using dichotomized data (longitudinal increase versus decrease in biomarker concentration), and the median PFS and OS intervals in each group were calculated. Statistical significance was determined using $p$-values, with a cut off of 0.025 being considered statistically significant in order to reduce the impact of multiple testing. More stringent adjustment for multiple comparisons was not considered due to the limited sample size. Analysis was carried out using $\mathrm{R}$ 3.5.0.

\section{Results}

\section{Patient characteristics}

Between October 2011 and November 2013, 20 patients were recruited to the study. Patient demographics are shown in Table 1. The mean age of participants was 69 years and the majority were male (85\%). During the study, the best radiological response to chemotherapy included: 12 patients (60\%) had RECIST complete or partial response (CR/PR), 2 patients (10\%) had RECIST stable disease (SD) and 6 patients (30\%) had disease progression. Across the entire cohort, the median PFS and OS were 8.7 and 17.3 months, respectively. Twelve

Table 1 Pre-treatment patient demographics

\begin{tabular}{ll}
\hline Patient demographic & Value \\
\hline Total patients & 20 \\
Sex: number (percentage) & $17(85 \%)$ \\
Male & $3(15 \%)$ \\
Female & \\
Age: (years) & 69 \\
Mean & $58-80$ \\
Range & \\
WHO performance status: number (percentage) & $7(35 \%)$ \\
0 & $12(60 \%)$ \\
1 & $1(5 \%)$ \\
2 & \\
Pre-treatment CEA ( $\mu \mathrm{g} / \mathrm{L}):$ & 384 \\
Mean & $3-2897$ \\
Range & \\
Pre-treatment LDH (IU/L): & 2279 \\
Mean & $45-11,346$ \\
Range & \\
Chemotherapy regimen: number (percentage) & $18(90 \%)$ \\
Oxaliplatin and 5FU & $2(10 \%)$ \\
\hline Oxaliplatin and capecitabine &
\end{tabular}


Table 2 Significant changes in circulating and imaging biomarkers from pre-treatment to cycle 2 day 2

\begin{tabular}{|c|c|c|c|}
\hline Biomarker type & Biomarker name & Mean difference from pre-treatment to C2D2 [95\% Cl] & $p$-value \\
\hline \multirow[t]{12}{*}{ Circulating } & VEGF-C & $-0.932[-1.333,-0.531]$ & 0.0002 \\
\hline & FGFb & $-0.866[-1.262,-0.469]$ & 0.0003 \\
\hline & VEGF-A & $-0.788[-1.16,-0.415]$ & 0.0004 \\
\hline & M65 & $-0.57[-0.845,-0.296]$ & 0.0004 \\
\hline & Ang2 & $-0.722[-1.084,-0.36]$ & 0.0006 \\
\hline & Ang1 & $-0.723[-1.101,-0.345]$ & 0.0009 \\
\hline & VEGF-D & $-0.316[-0.489,-0.143]$ & 0.0014 \\
\hline & CTCs & $-1.313[-2.074,-0.552]$ & 0.0021 \\
\hline & PDGFbb & $-0.44[-0.74--0.14]$ & 0.0070 \\
\hline & IL8 & $-0.47[-0.82--0.12]$ & 0.0124 \\
\hline & VCAM-1 & $0.32[0.07-0.58]$ & 0.0149 \\
\hline & E-selectin & $-0.3[-0.54--0.06]$ & 0.0183 \\
\hline \multirow[t]{6}{*}{ Imaging } & WTV $\left(\mathrm{mm}^{3}\right)$ & $-0.523[-0.751,-0.295]$ & 0.0002 \\
\hline & $\operatorname{ETV}\left(\mathrm{mm}^{3}\right)$ & $-0.56[-0.813,-0.307]$ & 0.0003 \\
\hline & $\mathrm{T} 1$ (ms) & $-0.144[-0.217,-0.071]$ & 0.0008 \\
\hline & $\mathrm{ADC}\left(\times 10^{-3} \mathrm{~mm} 2 / \mathrm{s}\right)$ & $0.01[0.00-0.02]$ & 0.0170 \\
\hline & $v_{e}$ & $0.04[0.01-0.07]$ & 0.0254 \\
\hline & $K^{\text {trans }}(/ \min )$ & $3.38[0.05-6.70]$ & $0.0469 *$ \\
\hline
\end{tabular}

Biomarker measurements were all log2 transformed, except ADC and $K^{\text {trans }}$ which were multiplied by 100, and iAUC, $v_{e}, v_{p}$ and EF which were not changed. Analysed using paired Student's t-test to assess for statistical significance

All circulating biomarkers measured in $\mathrm{pg} / \mathrm{ml}$. Imaging biomarkers are stated. $v_{\mathrm{e}}$ has no units

*Not statistically significant based on study cut-off of $p$ value $<0.025$

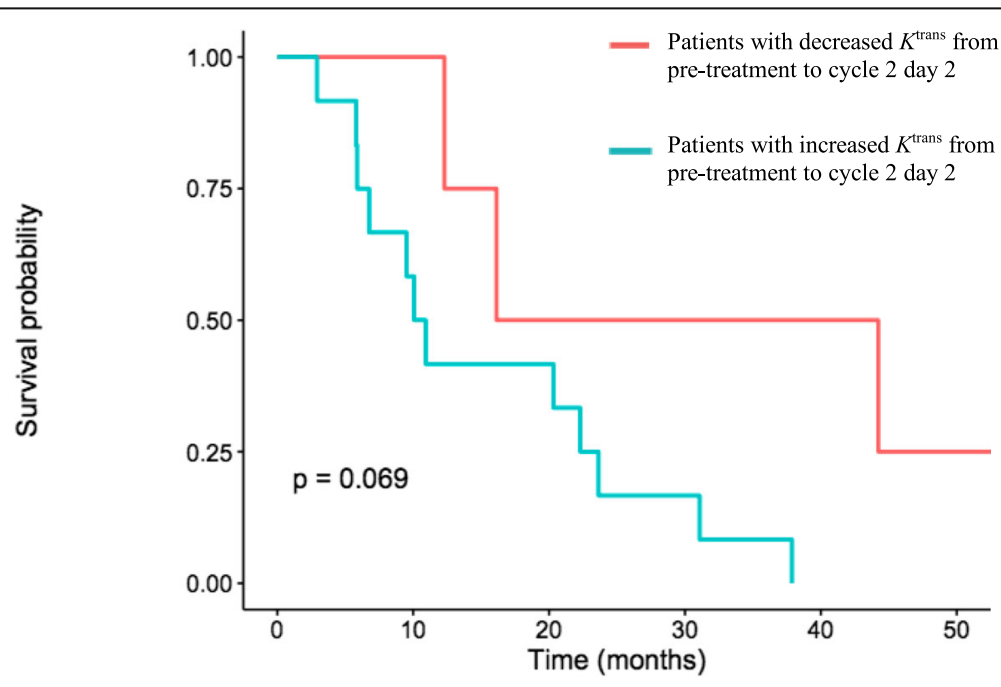

Number at risk

\begin{tabular}{|c|c|c|c|c|c|c|}
\hline \multirow[t]{2}{*}{ 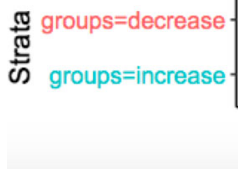 } & $\begin{array}{c}4 \\
12\end{array}$ & $\begin{array}{l}4 \\
7\end{array}$ & $\begin{array}{l}2 \\
5\end{array}$ & $\begin{array}{l}2 \\
2\end{array}$ & $\begin{array}{l}2 \\
0\end{array}$ & $\begin{array}{l}1 \\
0\end{array}$ \\
\hline & 0 & 10 & $\begin{array}{c}20 \\
\text { Tim }\end{array}$ & $\begin{array}{c}30 \\
\text { ths) }\end{array}$ & 40 & 50 \\
\hline
\end{tabular}

Fig. 1 Kaplan Meier Curve to show overall survival for patients with an increase in Ktrans from pre-treatment to cycle 2 day 2 compared with patients with a decrease in Ktrans from pretreatment to cycle 2 day 2 
patients completed all scanning protocols at chemotherapy cycle 6 and attrition occurred due to falling performance status throughout the trial. The imaging protocols were well tolerated and provided repeatable results.

\section{Pre-treatment biomarkers}

Pre-treatment characteristics including age, WHO performance status and pre-treatment concentrations of CEA and LDH were not associated with PFS or OS. Pretreatment, CTCs were detectable in all 20 patients. The mean number of CTCs was 4 per $7.5 \mathrm{ml}$ of blood.

Evaluation of the association between survival outcomes and pre-treatment concentrations of circulating biomarkers showed that lower concentrations of Ang2 (HR 0.41, 95\%CI 0.19-0.86, $p=0.019$ ) and VEGF-A (HR $0.41,95 \%$ CI $0.19-0.87, p=0.021)$ were associated with a significantly reduced PFS. No other pre-treatment biomarkers were found to be associated with PFS or OS.

\section{Biomarkers on treatment}

Data showed that the plasma concentration of most circulating angiogenesis-related biomarkers reduced from pre-treatment to cycle 2 day 2 , with significant reductions in Ang1, Ang2, VEGF-A, VEGF-C, and VEGF-D (Table 2). VCAM-1 was the only circulating biomarker to significantly increase $(p=0.0194)$. The increase of VCAM-1 and decrease of all other circulating biomarkers from pre-treatment to cycle 2 day 2 was not associated with PFS or OS.

The mean number of CTCs significantly reduced from pre-treatment to cycle 2 day $2(p=0.0021)$. A higher number of CTCs at cycle 2 day 2 was associated with significantly worse OS (HR 2.82, 95\%CI 1.3-6.1, $p=0.008$ ).

MRI data showed that WTV, enhancing tumour volume (ETV) and T1 decreased significantly from pretreatment to cycle 2 day $2(\mathrm{p}=0.002, p=0003$ and $p=$ 0008 , respectively). The ADC significantly increased from pre-treatment to cycle 2 day $2(p=0.017)$ (Table 2). However, none of these parameters were associated with PFS or OS. On average, there was an increase in $K^{\text {trans }}$ from pre-treatment to cycle 2 day 2 . In those patients whose $K^{\text {trans }}$ increased at cycle 2 day 2 , there was a significantly worse OS outcome when compared to those patients whose $K^{\text {trans }}$ did not increase at cycle 2 day 2 (HR 1.099, 95\%CI 1.01-1.20, $p=0.025$ ) (Fig. 1).

All patients had an increased CEA concentration from pre-treatment to cycle 2, but this was not associated

\section{A}

Correlation network before treatment

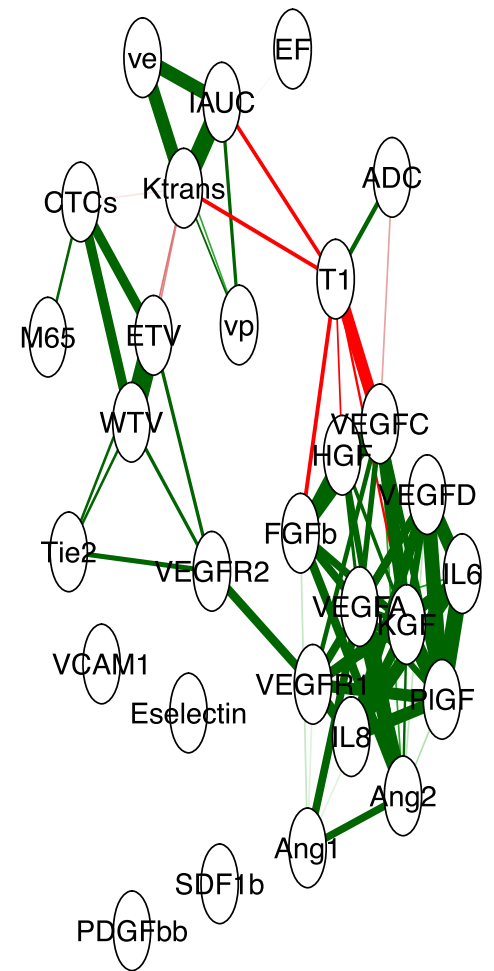

B

Correlation network at cycle 2 day 2

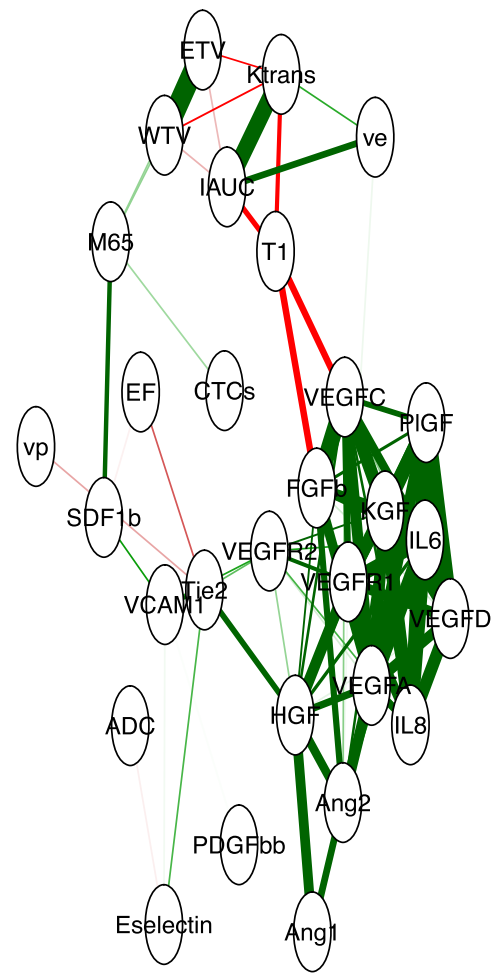

Fig. 2 Correlation network to compare correlation between biomarkers at pre-treatment and at cycle 2 day 2. Correlation networks show the positive (green) and negative (red) correlations between biomarkers. The plot shows that both at pre-treatment and at cycle 2 day 2 the angiogenic biomarkers cluster closely together in a network 
with PFS nor OS (PFS: $p=0.521$, OS: $p=0.638$ ). There was no significant difference between the mean increase in CEA concentration between patients with an increased $K^{\text {trans }}$ and those with a decreased $K^{\text {trans }}(6.79$ [95\% CI 6.17-7.36] versus 6.51 [95\% CI 6.21-6.81], respectively, $p=0.43)$.

The correlation network analysis showed that, across all patients, the change in the circulating concentrations of angiogenesis-related proteins in response to chemotherapy was similar. The interaction between these proteins is undisturbed by cytotoxic chemotherapy, shown by the close clustering of angiogenic biomarkers both at pre-treatment and at cycle 2 day 2 (Fig. 2).

See Supplementary Information for the full data set.

\section{Discussion}

In this study we have investigated the impact of cytotoxic chemotherapy on circulating proteins and CTCs as surrogate markers of tumour vasculature and cellularity, in parallel with MRI, to document changes in tumour perfusion and tumour volume. This study was limited by the small numbers of participants and so should be regarded as exploratory only.

A key finding of this study was that an increase in $K^{\text {trans }}$ at cycle 2 day 2 was associated with a worse OS. In contrast, trials of anti-angiogenic or vascular disrupting agents show near universal early decrease in $K^{\text {trans }}[19]$. This is considered to reflect reduction in either perfusion or permeability of tumour blood vessels, or a combination of the two processes, rather than a systemic effect. The process by which $K^{\text {trans }}$ changes with a cytotoxic chemotherapy regimen is more complex and indirect. It is possible that is that in these patients, the tumour adapts to chemotherapy by increasing in its vasculature. Another possibility is that these patients have tumours which partly respond to chemotherapy, as shown by the reduction in WTV, but the remaining tumour mass consists of well-perfused chemotherapyresistant tissue. This process could be explained by a "healing response" to chemotherapy, causing an increase in tumour vascular function and reflected by an increased $K^{\text {trans }}$ value. As the network analysis does not describe changes that are associated with individual biomarkers in isolation, but rather global reductions in angiogenesis biomarkers, the implication is that there is not a dynamic biological response to chemotherapy. Thus, chemotherapy is killing sensitive tumour cell populations leaving behind more resistant clones; characterised here with high $K^{\text {trans }}$. However, since massive cell death of both tumour and non-tumour cells resulting from chemotherapy can lead to the release of damage associated molecular pattern (DAMPs) proteins, it is possible that any resultant systemic inflammatory response could lead to increase in vasculature permeability, detected an increased $K^{\text {trans }}$.

Interestingly, the standard of care marker of treatment response, CEA concentration, increased in all patients from pre-treatment to cycle 2. As mentioned, there was no significant difference between the mean increase in CEA concentration between patients with an increased $K^{\text {trans }}$ and those with a decreased $K^{\text {trans }}$. This suggests that $K^{\text {trans }}$ could be more useful than CEA to predict poorer responses to cytotoxic chemotherapy. This finding may also direct earlier introduction of antiangiogenic agents, such as bevacizumab.

This study also shows an association between lower pre-treatment circulating concentrations of Ang2 and VEGF-A and worse PFS. There is inconsistency in the value of pre-treatment biomarkers of angiogenesis reported in the literature [29-31]. Results differ between

Table 3 Correlation between pre-treatment circulating angiogenesis-related biomarkers and survival outcomes in studies which recruited patients with metastatic colorectal cancer [22-24]

\begin{tabular}{|c|c|c|c|c|}
\hline Study Title & $\begin{array}{l}\text { No. of } \\
\text { patients }\end{array}$ & Treatment received & $\begin{array}{l}\text { Pre- } \\
\text { treatment } \\
\text { biomarker }\end{array}$ & Correlation with survival outcomes \\
\hline \multirow{5}{*}{$\begin{array}{l}\text { Prognostic/predictive value of } 207 \text { serum factors in } \\
\text { colorectal cancer treated with cediranib and/or } \\
\text { chemotherapy }^{a}[22]\end{array}$} & \multirow[t]{5}{*}{582} & \multirow{5}{*}{$\begin{array}{l}\text { FOLFOX or CAPOX } \\
\text { chemotherapy }+ \\
\text { cediranib/placebo }\end{array}$} & VEGF-D & \multirow{4}{*}{$\begin{array}{l}\text { Low pre-treatment concentration corre- } \\
\text { lated with improved PFS and OS regard- } \\
\text { less of treatment received }\end{array}$} \\
\hline & & & VEGFR-1 & \\
\hline & & & VEGFR-3 & \\
\hline & & & Tie-2 & \\
\hline & & & Ang2 & No correlation \\
\hline $\begin{array}{l}\text { Changes in circulating VEGF levels in relation to clinical } \\
\text { response during chemotherapy for metastatic cancer [23] }\end{array}$ & 90 & Camptothecin & VEGF-165 & $\begin{array}{l}\text { Patients with high pre-treatment concen- } \\
\text { tration were more likely to have progres- } \\
\text { sive disease during treatment }\end{array}$ \\
\hline $\begin{array}{l}\text { Phase II Trial of Infusional Fluorouracil, Irinotecan, and } \\
\text { Bevacizumab for Metastatic Colorectal Cancer: Efficacy }\end{array}$ & 43 & $\begin{array}{l}\text { FOLFIRI }+ \\
\text { bevacizumab }\end{array}$ & VEGF-2 & No correlation \\
\hline
\end{tabular}


studies (Table 3) but are also difficult to compare due to different angiogenesis-related proteins investigated and methods of sampling/analysis used. Hence, our study focussed on the significance of dynamic response to treatment rather than pre-treatment concentrations.

\section{Conclusion}

In conclusion, the response to cytotoxic chemotherapy treatment in patients with colorectal cancer with liver metastases showed a maintained robust relationship between angiogenic biomarkers. In some patients, poor outcome was associated with the early detection of wellperfused tissue in smaller tumours suggesting that chemotherapy was unable to kill the remaining component of a tumour, presumably because of increased clearance of cytotoxic agents. These findings identify a group of patients whose tumour does not respond well to traditional cytotoxic chemotherapy alone and who might benefit from early addition of molecularly targeted therapies.

\section{Abbreviations \\ 5-FU: Fluorouracil; ADC: Apparent diffusion coefficient; Ang: Angiopoetin; BSA: Body surface area; CAPOX: Capecitabine plus oxaliplatin; \\ CEA: Carcinoembryonic antigen; Cl: Confidence interval; CK18: Cytokeratin-18; $\mathrm{CT}$ : Computed tomography; CTC: Circulating tumour cells; CR: Complete response; DAMPs: Damage associated molecular pattern; DCE-MRI: Dynamic contrast-enhanced magnetic resonance imaging; DWI-MRI: Diffusion weighted magnetic resonance imaging; EGFR: Epidermal growth factor receptors; ETV: Enhancing tumour volume; FGFb: Basic fibroblastic growth factor; FOLFIRI: Fluorouracil/folinic acid plus irinotecan; FOLFOX: Fluorouracil/ folinic acid plus oxaliplatin; HGF: Hepatocyte growth factor; HR: Hazard ratio; iAUC: Incremental area under the curve; IL8: Interleukin 8; KGF: Keratinocyte growth factor; $K^{\text {trans }}$ : Endothelial contrast agent transfer coefficient; LDH: Lactate dehydrogenase; MRI: Magnetic resonance imaging; NHS: National Health Service; OS: Overall survival; PDGFbb: Platelet derived growth factor beta; PFS: Progression-free survival; PIGF: Placental growth factor; PR: Partial response; RECIST: Response evaluation criteria in solid tumours; ROI: Regions of interest; SD: Stable disease; SDF1b: Stromal cell- derived factor 1; ULN: Upper limit of normal; VCAM1: Vascular cell adhesion molecule 1; $v_{e}$ : Fractional extravascular extracellular volume; VEGF: Vascular endothelial growth factor; VEGFR: Vascular endothelial growth factor receptor; $v_{p}$ : Fractional blood plasma volume; WHO: World Health Organization; WTV: Whole tumour volume}

\section{Supplementary Information}

The online version contains supplementary material available at https://doi. org/10.1186/s12885-021-08097-9.

\section{Additional file 1.}

\section{Acknowledgements}

The authors would like to thank the patients involved in this study and their families. We gratefully acknowledge the support funding received from Cancer Research UK, AstraZeneca Pharmaceuticals and Manchester NIHR Biomedical Research Centre and the support of the host institutions, CRUK Manchester Institute, and the Wolfson Molecular Imaging Centre, Manchester. We would like to thank The Systemic Therapy Research Group at The Christie NHS Foundation Trust for their assistance with preparation of the manuscript. The sponsor for this study was The Christie Hospital NHS Foundation Trust.

\section{Authors' contributions}

RDM1 and DS contributed equally as 1st authors to the interpretation of results and write up of the manuscript. GCJ oversaw the entire project from conduct of the study, interpretation of data and manuscript write up. TD and CZ performed statistical analysis of the data and reviewed the finished manuscript. DS, NM, MS and SM conducted the trial and oversaw recruitment, patient management, imaging studies and sample collection. $A B$ and $K M$ conducted the ELISA analyses throughout the project. CD is director of the circulating biomarkers laboratory and oversaw the conduct and analysis of all ELISAs. AJ, JPBO'C, and GJMP oversaw the advanced imaging, from acquisition, standardization, data acquisition, analysis, and Q.C. standards. RAL, YW and SC were responsible for application of DCE-MRI protocols and definition of regions of interest. RDM2, JPBO'C, GJMP and $A B$ all had significant input in substantive revisions of the manuscript and all authors have approved the final manuscript.

\section{Funding}

Funding received from Cancer Research UK, Manchester NIHR Biomedical Research Centre and AstraZeneca Pharmaceuticals. The funding bodies played no role in the design of the study and collection, analysis, and interpretation of data and in writing the manuscript.

\section{Availability of data and materials}

The datasets used and/or analysed during the current study are available from the corresponding author on reasonable request.

\section{Declarations}

\section{Ethics approval and consent to participate}

This trial was performed with local ethics committee approval from the NRES Committee North West - Liverpool East, under the NHS Health Research Authority (11/NW/0118) [32]. Local research and development department approval was obtained and laboratory work was carried out in accordance with the principles of Good Clinical Laboratory Practice (World Health Organization 2009). All patients gave written informed consent to participate in the study.

\section{Consent for publication}

Not applicable.

\section{Competing interests}

GJMP is a director and shareholder in Bioxydyn Limited, a company with an interest in advanced MRI biomarkers.

No other competing interests

\section{Author details}

${ }^{1}$ Christie NHS Foundation Trust, Wilmslow Road, Withington, Manchester M20 4BX, UK. ${ }^{2}$ The Clatterbridge Cancer Centre NHS Foundation Trust, Wirral, UK. ${ }^{3}$ Cancer Research UK Manchester Institute Cancer Biomarker Centre, University of Manchester, Alderley Park, Macclesfield, UK. ${ }^{4}$ Division of Cancer Sciences, School of Medicine, University of Manchester, Manchester, UK. ${ }^{5}$ Division of Informatics, Imaging and Data Sciences, School of Health Sciences, University of Manchester, Manchester, UK. 'Bioxydyn Limited, Manchester, UK. ${ }^{7}$ Department of Computer Science, Centre for Medical Image Computing, University College London, London, UK.

Received: 19 July 2020 Accepted: 24 March 2021

Published online: 01 April 2021

\section{References}

1. UK CR. Bowel Cancer statistics [internet]. 2016. Available from: https://www. cancerresearchuk.org/health-professional/cancer-statistics/statistics-by-ca ncer-type/bowel-cancer

2. Office for National Statistics. Cancer survival in England - adults diagnosed [Internet]. Available from: https://www.ons.gov.uk/peoplepopulationa ndcommunity/healthandsocialcare/conditionsanddiseases/datasets/ca ncersurvivalratescancersurvivalinenglandadultsdiagnosed.

3. Van Cutsem E, Cervantes A, Adam R, Sobrero A, Van Krieken JH, Aderka D, et al. ESMO consensus quidelines for the management of patients with metastatic colorectal cancer. Ann Oncol. 2016;27(8):1386-422. https://doi. 
org/10.1093/annonc/mdw235 Available from: https://inkinghub.elsevier. com/retrieve/pii/S0923753419347544.

4. National Comprehensive Cancer Network. NCCN clinical practice guidelines in oncology. Colon Cancer. 2019.

5. Hurwitz H, Fehrenbacher L, Novotny W, Cartwright T, Hainsworth J, Heim W, et al. Bevacizumab plus Irinotecan, fluorouracil, and Leucovorin for metastatic colorectal Cancer. N Engl J Med. 2004;350(23):2335-42. https:// doi.org/10.1056/NEJMoa032691.

6. Cunningham D, Lang I, Marcuello E, Lorusso V, Ocvirk J, Shin DB, et al. Bevacizumab plus capecitabine versus capecitabine alone in elderly patients with previously untreated metastatic colorectal cancer (AVEX): an openlabel, randomised phase 3 trial. Lancet Oncol. 2013;14(11):1077-85. Available from: https://linkinghub.elsevier.com/retrieve/pii/S1470204513701542. https://doi.org/10.1016/S1470-2045(13)70154-2.

7. Kabbinavar F, Irl C, Zurlo A, Hurwitz H. Bevacizumab improves the overall and progression-free survival of patients with metastatic colorectal Cancer treated with 5-fluorouracil-based regimens irrespective of baseline risk. Oncology. 2008;75(3-4):215-23. Available from: https:/www.karger.com/A rticle/FullText/163850. https://doi.org/10.1159/000163850.

8. Saltz LB, Clarke S, Díaz-Rubio E, Scheithauer W, Figer A, Wong R, et al. Bevacizumab in combination with oxaliplatin-based chemotherapy as firstline therapy in metastatic colorectal cancer: a randomized phase III study. J Clin Oncol. 2008;26(12):2013-9. https://doi.org/10.1200/JCO.2007.14.9930.

9. Cremolini C, Loupakis F, Antoniotti C, Lupi C, Sensi E, Lonardi S, et al. FOLFOXIRI plus bevacizumab versus FOLFIRI plus bevacizumab as first-line treatment of patients with metastatic colorectal cancer: updated overall survival and molecular subgroup analyses of the open-label, phase 3 TRIBE study. Lancet Oncol. 2015;16(13):1306-15. Available from: https://linkinghub. elsevier.com/retrieve/pii/S1470204515001229. https://doi.org/10.1016/S14 70-2045(15)00122-9.

10. Van Cutsem E, Nowacki M, Lang I, Cascinu S, Shchepotin I, Maurel J, et al. Randomized phase III study of irinotecan and 5-FU/FA with or without cetuximab in the first-line treatment of patients with metastatic colorectal cancer (mCRC): The CRYSTAL trial. J Clin Oncol. 2007;25(18_suppl):4000. https://doi.org/10.1200/jco.2007.25.18_suppl.4000.

11. Van Cutsem E, Köhne C-H, Hitre E, Zaluski J, Chang Chien C-R, Makhson A, et al. Cetuximab and chemotherapy as initial treatment for metastatic colorectal cancer. N Engl J Med. 2009;360(14):1408-17. https://doi.org/10.1 056/NEJMoa0805019.

12. Douillard J-Y, Siena S, Cassidy J, Tabernero J, Burkes R, Barugel M, et al. Randomized, phase III trial of panitumumab with Infusional fluorouracil, leucovorin, and oxaliplatin (FOLFOX4) versus FOLFOX4 alone as first-line treatment in patients with previously untreated metastatic colorectal cancer: the PRIME study. J Clin Oncol. 2010;28(31):4697-705. https://doi.org/10.1200/ JCO.2009.27.4860.

13. Tebbutt NC, Wilson K, Gebski VJ, Cummins MM, Zannino D, van Hazel GA, et al. Capecitabine, bevacizumab, and mitomycin in first-line treatment of metastatic colorectal cancer: results of the Australasian gastrointestinal trials group randomized phase III MAX study. J Clin Oncol. 2010;28(19):3191-8. https://doi.org/10.1200/JCO.2009.27.7723.

14. Greystoke A, Dean E, Saunders MP, Cummings J, Hughes A, Ranson M, et al. Multi-level evidence that circulating CK18 is a biomarker of tumour burden in colorectal cancertle. Br J Cancer. 2012;107(9):1518-24. https://doi.org/10.1 038/bjc.2012.416.

15. Scott LC, Evans TRJ, Cassidy J, Harden S, Paul J, Ullah R, et al. Cytokeratin 18 in plasma of patients with gastrointestinal adenocarcinoma as a biomarker of tumour response. Br J Cancer. 2009;101(3):410-7. Available from: http:// www.nature.com/articles/6605175. https://doi.org/10.1038/sj.bjc.6605175.

16. Douillard J-Y, Oliner KS, Siena S, Tabernero J, Burkes R, Barugel M, et al. Panitumumab-FOLFOX4 treatment and RAS mutations in colorectal cancer. N Engl J Med. 2013;369(11):1023-34. https://doi.org/10.1056/NEJMoa13052 75.

17. Ciardiello F, Lenz H-J, Kohne C-H, Heinemann V, Tejpar S, Melezinek I, et al. Treatment outcome according to tumor RAS mutation status in CRYSTAL study patients with metastatic colorectal cancer ( $m C R C$ ) randomized to FOLFIRI with/without cetuximab. J Clin Oncol. 2014;32(15_suppl):3506. https://doi.org/10.1200/jco.2014.32.15_suppl.3506.

18. Bokemeyer C, Kohne C-H, Ciardiello F, Lenz H-J, Heinemann V, Klinkhardt U, et al. Treatment outcome according to tumor RAS mutation status in OPUS study patients with metastatic colorectal cancer (mCRC) randomized to
FOLFOX4 with/without cetuximab. J Clin Oncol. 2014;32(15_suppl):3505. https://doi.org/10.1200/jco.2014.32.15 suppl.3505.

19. O'Connor JPB, Jackson A, Parker GJM, Roberts C, Jayson GC. Dynamic contrast-enhanced MRI in clinical trials of antivascular therapies. Nat Rev Clin Oncol. 2012;9(3):167-77. Available from: http://www.nature.com/a rticles/nrclinonc.2012.2. https://doi.org/10.1038/nrclinonc.2012.2.

20. Anzidei M, Napoli A, Zaccagna F, Cartocci G, Saba L, Menichini G, et al. Liver metastases from colorectal cancer treated with conventional and antiangiogenetic chemotherapy. J Comput Assist Tomogr. 2011;35(6):690-6. Available from: https://insights.ovid.com/crossref?an=00004728-20111100000006. https://doi.org/10.1097/RCT.0b013e318230d905.

21. O'Connor JPB, Carano RAD, Clamp AR, Ross J, Ho CCK, Jackson A, et al. Quantifying Antivascular effects of monoclonal antibodies to vascular endothelial growth factor: insights from imaging. Clin Cancer Res. 2009; 15(21):6674-82. https://doi.org/10.1158/1078-0432.CCR-09-0731.

22. Koh D-M, Scurr E, Collins D, Kanber B, Norman A, Leach MO, et al. Predicting response of colorectal hepatic metastasis: value of pretreatment apparent diffusion coefficients. Am J Roentgenol. 2007;188(4):1001-8. https://doi.org/1 0.2214/AJR.06.0601.

23. Jayson GC, Zhou C, Backen A, Horsley L, Marti-Marti K, Shaw D, et al. Plasma Tie2 is a tumor vascular response biomarker for VEGF inhibitors in metastatic colorectal cancer. Nat Commun. 2018;9(1):4672 Available from: http://www.nature.com/articles/s41467-018-07174-1.

24. Zhou C, Clamp A, Backen A, Berzuini C, Renehan A, Banks RE, et al. Systematic analysis of circulating soluble angiogenesis-associated proteins in ICON7 identifies Tie2 as a biomarker of vascular progression on bevacizumab. Br J Cancer. 2016;115(2):228-35. Available from: http://www. nature.com/articles/bjc2016194. https://doi.org/10.1038/bjc.2016.194.

25. Backen A, Renehan AG, Clamp AR, Berzuini C, Zhou C, Oza A, et al. The combination of circulating Ang1 and Tie2 levels predicts progression-free survival advantage in Bevacizumab-treated patients with ovarian Cancer. Clin Cancer Res. 2014;20(17):4549-58. https://doi.org/10.1158/1078-0432. CCR-13-3248.

26. Eisenhauer EA, Therasse P, Bogaerts J, Schwartz LH, Sargent D, Ford R, et al. New response evaluation criteria in solid tumours: revised RECIST guideline (version 1.1). Eur J Cancer. 2009;45(2):228-47 Available from: https:// linkinghub.elsevier.com/retrieve/pii/S0959804908008733.

27. Li G, Wang Z, Xu J, Wu H, Cai S, He Y. The prognostic value of lactate dehydrogenase levels in colorectal cancer: a meta-analysis. BMC Cancer. 2016;16(1):249. https://doi.org/10.1186/s12885-016-2276-3.

28. Duffy M, van Dalen A, Haglund C, Hansson L, Klapdor R, Lamerz R, et al. Clinical utility of biochemical markers in colorectal cancer. Eur J Cancer. 2003;39(6):718-27. Available from: https://linkinghub.elsevier.com/retrieve/ pii/S0959804902008110. https://doi.org/10.1016/S0959-8049(02)00811-0.

29. Spencer SKM, Pommier AJC, Morgan SR, Barry ST, Robertson JD, Hoff PM, et al. Prognostic/predictive value of 207 serum factors in colorectal cancer treated with cediranib and/or chemotherapy. Br J Cancer. 2013;109(11): 2765-73. Available from: http://www.nature.com/articles/bjc2013649. https://doi.org/10.1038/bjc.2013.649.

30. Lissoni P, Rovelli F, Malugani F, Brivio F, Fumagalli L, Gardani GS. Changes in circulating VEGF levels in relation to clinical response during chemotherapy for metastatic cancer. Int J Biol Markers. 2003;18(2):152-5. Available from: http://www.biological-markers.com/article/changes-in-circulating-vegf-levelsin-relation-to-clinical-response-during-chemotherapy-for-metastatic-cancer-a rt005450. https://doi.org/10.1177/172460080301800209.

31. Kopetz S, Hoff PM, Morris JS, Wolff RA, Eng C, Glover KY, et al. Phase II trial of Infusional fluorouracil, Irinotecan, and Bevacizumab for metastatic colorectal cancer: efficacy and circulating angiogenic biomarkers associated with therapeutic resistance. J Clin Oncol. 2010;28(3):453-9. https://doi.org/1 $0.1200 / J C O .2009 .24 .8252$

32. UK Legislation. The medicines for human use (clinical trials) regulations 2004 [Internet]. 2004 [cited 2020 May 12]. Available from: http://www.legisla tion.gov.uk/uksi/2004/1031/pdfs/uksi_20041031_en.pdf.

\section{Publisher's Note}

Springer Nature remains neutral with regard to jurisdictional claims in published maps and institutional affiliations. 